\title{
TRANSFORMACIONES AGRÍCOLAS EN EL CONTEXTO PERIURBANO DE LA CIUDAD DE San Cristóbal de Las Casas, Chiapas
}

\author{
Agricultural Transformations in the Periurban Context of San Cristóbal de Las Casas City, Chiapas \\ Araceli Calderón-Cisneros \\ Lorena Soto-Pinto
}

Resumen: La ciudad de San Cristóbal de Las Casas ha crecido considerablemente en las últimas décadas abarcando las zonas rurales circundantes. El objetivo del presente trabajo consistió en identificar las transformaciones que la interacción urbano-rural imprime sobre la actividad agrícola de un área periurbana: el cerro Huitepec. Se revisó información documental y se levantaron 100 entrevistas abiertas y estructuradas entre población de las localidades rurales e informantes clave. Aunque ha perdido relevancia como fuente de ingresos económicos familiares, la agricultura y la cría de animales de traspatio aún tienen un papel significativo para el autoconsumo familiar; no obstante, un pequeño grupo de productores ha logrado articularse a un mercado de hortalizas orgánicas orientado a un sector de la población urbana, revitalizando su actividad agrícola.

Palabras clave: agricultura periurbana, nueva ruralidad, estudios periurbanos, mercados orgánicos.

Abstract: San Cristobal de Las Casas city has grown considerably in recent decades, covering the surrounding rural areas. The aim of this study was to identify transformations on the agricultural activity in the Huitepec (a nearby mountain) as a result of urban-rural interactions. Documentary information was reviewed, and 100 open and structured interviews were applied among people in the rural localities and key informants. Although it has lost relevance as a source of family income, agriculture and backyard animal production still have a significant role for family consumption. However, a small group of farmers has achieved to link with an organic vegetable market oriented towards a sector of urban population, revitalizing their agricultural activity.

Keywords: periurban agriculture, new rurality, periurban studies, organic markets.

Araceli Calderón Cisneros, doctora en ciencias por El Colegio de la Frontera Sur, México. Investigadora independiente. Temas de especialización: recursos naturales, género y medio ambiente, desarrollo sustentable. Correo electrónico: aracalcis@yahoo.com.mx.

Lorena Soto Pinto, doctora en ciencias por la Universidad Nacional Autónoma de México. Investigadora de El Colegio de la Frontera Sur, México. Temas de especialización: sistemas agroforestales y agroforestería comunitaria. Correo electrónico: 1soto@ecosur.mx.
Enviado a dictamen: 23 de mayo de 2013.

Aprobación: 20 de julio de 2013.

Revisiones: 1. 


\section{Introducción}

L os efectos del crecimiento urbano sobre los espacios rurales y naturales circundantes han sido analizados en numerosos trabajos que dan cuenta de las transformaciones de tipo social, económico y geográfico que ocurren en las áreas aledañas a las grandes ciudades, sobre todo del centro del país (Arias, 2009; Torres y Rodríguez, 2008; Benítez, 2006; Cruz, 2002; García, 2002; Ramírez, 2000; Aguilar y Escalona, 2000). ${ }^{1}$ Entre los cambios más relevantes se señalan: la transformación de las dinámicas familiares económico-productivas marcadas por la reducción de los ingresos agrícolas y la diversificación de fuentes de ingresos monetarios; los intensos procesos de cambio de uso de suelo y mercado de tierras con fines habitacionales que afectan en mayor medida a las tierras agrícolas; y los cambios culturales y sociales que se derivan de la integración de las dinámicas urbanas y rurales en un mismo espacio. Estas situaciones constituyen manifestaciones particulares de los cambios que, en general, ha experimentado el medio rural latinoamericano en las últimas décadas a partir de los procesos de globalización económica, y que han sido identificados por algunos autores como una "nueva ruralidad", en la que los referentes tradicionales de lo urbano y lo rural como espacios diferenciados y opuestos ya no tienen correspondencia con la realidad (Carton de Grammont, 2010; Lambí y Pérez, 2007; Kay, 2009). ${ }^{2}$ En términos generales, se distinguen tres tipos de transformaciones: a) territoriales, a partir del cambio en la valoración de los espacios rurales y la emergencia de nuevas formas de organización del espacio; b) ocupacionales, debido al cambio del tipo y el peso de las actividades primarias, secundarias y terciarias en la economía rural; y c) culturales, ante el cambio en los patrones del conocimiento y valores de las poblaciones rurales (Gómez, 2002).

Los espacios periurbanos constituyen una de las manifestaciones específicas de la nueva ruralidad. El concepto de periurbano se utiliza para hacer referencia tanto a un espacio geográfico, como a los procesos sociales que ocurren en él (Ruiz y Delgado, 2008; Avila, 2009; Morello et al., 2003). Desde una perspectiva geográfica, lo periurbano puede manifestarse territorialmente, en el caso de las grandes ciudades y conglomerados urbanos, en la conformación de coronas o espacios periféricos concéntricos entre los que permanecen intersticios rurales (Ávila, 2009; Ruiz y Delgado, 2008). Sin embargo, otros autores se refieren al espacio periurbano como al territorio contiguo a la ciudad en el que se producen cambios ambientales permanentes derivados directamente de su crecimiento (Morello et al., 2003), independientemente de su tamaño. Socialmente, los espacios periurbanos presentan dinámicas complejas y conflictivas ante los cambios sociales, económicos y culturales que ocurren, sobre todo en los asentamientos rurales, al integrarse a la ciudad (Arias, 2009; Ávila, 2009); por otra parte, Banzo (2005) considera que en este territorio emerge una forma de vida vinculada a ese espacio que incluye valores de sustentabilidad ambiental.

Desde la óptica del desarrollo rural, uno de los aspectos que resulta de suma importancia en el espacio periurbano es la cuestión de la tierra y la agricultura por los conflictos que surgen como consecuencia del consumo del espacio para uso urbano (Ávila, 2004). Sin embargo, paradójicamente, en las ciudades los sistemas agrícolas y alimentarios han estado presentes históricamente, y en algunas regiones del mundo incluso tienden a incrementarse como resultado de la migración del campo a las ciudades, la creciente pobreza urbana y el incremento en el costo de los alimentos (Bryld, 2002; Burns, 2000; Pothukuchi y Kaufman, 1999).

Se considera como agricultura urbana aquella producción agrícola y animal que ocurre dentro de las ciudades y su periferia y que recibe influencia directa de la ciudad, de modo que sus procesos productivos están íntimamente ligados a la población urbana (Altieri et al., 1999). Aunque no existe una claridad sobre la magnitud de este tipo de agricultura a nivel mundial, se ha observado que es una actividad muy relevante en los países en vías de desarrollo involucra entre el 11\% y el 69\% de la población urbana de algunos países - como fuente de autoconsumo de alimentos $y$, en menor medida, como fuente de 
ingresos económicos para las familias urbanas pobres (Zezza y Tasciotti, 2010). En este sentido, la agricultura urbana puede tener un papel clave en la producción alimentaria local y para el desarrollo sustentable en las ciudades (Ávila, 2004; Altieri et al., 1999), por lo que en algunos países se han aplicado políticas que la favorecen; sin embargo, en términos generales la agricultura urbana es una actividad que tiende a ser marginalizada o ilegal, ya sea implícita o explícitamente, ante las políticas diferenciadas de lo que es correcto para el medio urbano y el rural (Bryld, 2002). Es por ello que el reconocimiento de la nueva configuración de la vida rural y sus conexiones con los centros urbanos permite que ambos espacios sean abordados como un conjunto interdependiente, lo cual puede tener implicaciones en términos de políticas públicas y propuestas locales de desarrollo.

Los territorios periurbanos resultan particularmente interesantes para el análisis de las transformaciones que ocurren a partir de la mayor presencia de las dinámicas urbanas en la vida rural, y particularmente en la actividad agrícola. En México se ha observado que el crecimiento urbano ha provocado en su periferia la pérdida de grandes extensiones de tierras de calidad agrícola y forestal, al tiempo que se favorece una reorientación de la función agrícola como proveedora de alimentos para las familias rurales hacia la producción de alimentos y otros bienes destinados a los centros urbanos (Torres y Rodríguez, 2008; Benítez, 2006; García, 2002; Salazar, 2000). Sin embargo, la mayor parte de estos estudios se ha realizado en las grandes ciudades del centro del país, mientras que se ha puesto poca atención a las ciudades medias y pequeñas a pesar de que albergan más de la mitad de la población urbana nacional y de que tendrán un fuerte crecimiento poblacional en el futuro. Estas ciudades juegan un papel relevante como articuladoras territoriales entre las grandes ciudades y el medio rural, y, por lo tanto, son referentes obligados en la búsqueda de un desarrollo sustentable y territorial (Bellet y Llop, 2002).

En el sureste del país se presentan condiciones particulares de urbanización ya que la población tiende a concentrarse en pocas ciudades, pero, al mismo tiempo, un alto porcentaje se atomiza en numerosas localidades rurales distribuidas en el territorio, por lo que cabe preguntarse cómo se manifiestan los fenómenos periurbanos en estos espacios. Chiapas es uno de los estados que presenta mayor proporción de población rural en el país dado que un $67 \%$ habita en localidades rurales y semirurales con menos de 15000 habitantes, y sólo tres localidades, que concentran el $20 \%$ de la población, superan los 100000 habitantes (INEGI, 2010).

San Cristóbal de Las Casas es una ciudad media que tiene una población de casi 160000 habitantes; se ha caracterizado por presentar un rápido crecimiento tanto de su población como de la superficie que ocupa (ver mapa 1), especialmente desde 1970, cuando apenas contaba con unos 26000 habitantes (INEGI, 2010; CONAPO, 2005; García, 2005). Actualmente, San Cristóbal concentra el 85\% de la población municipal, y el porcentaje restante se distribuye en 96 localidades rurales situadas a su alrededor. Al mismo tiempo, el municipio es el centro rector de la región Altos tsotsiltseltal que incluye otros 16 municipios, principalmente de población indígena, los cuales presentan muy alto grado de marginación. Lo anterior implica una gran presión para la ciudad como referente regional en la búsqueda de oportunidades de desarrollo, y ha propiciado un crecimiento caótico e irregular a partir de flujos de inmigración regional que se ven acentuados en periodos de conflicto político o social, tales como las expulsiones religiosas de los años ochenta en los municipios vecinos, o la rebelión zapatista en 1994; ello ha ocasionado que gran parte del espacio urbanizado de la ciudad se haya ocupado por la vía ilegal (Velázquez, 2004). A este crecimiento por inmigración regional se ha sumado una gran cantidad de personas originarias de otros estados y del extranjero, que radican de manera permanente o temporal en esta ciudad de gran atractivo turístico.

San Cristóbal se ubica en una cuenca cerrada a 2200 metros sobre el nivel del mar, rodeada por montañas que presentan bosques de encino y pino, fragmentos de bosque mesófilo y humedales de montaña que son considerados ecosistemas prioritarios para conservación 
(Arriaga et al., 2000); asimismo, los sistemas agrícolas tradicionales han formado parte de las estrategias de vida de la población rural de la región (Morales et al., en prensa). La necesidad de vivienda, junto con la falta de regulación municipal y los fuertes intereses económicos que se derivan de la demanda de espacio habitacional, están propiciando la pérdida de áreas boscosas y agrícolas en los alrededores de la ciudad, así como de los humedales naturales situados en el valle, que poco a poco se incorporan al uso urbano. En su crecimiento, la superficie urbana también se acerca a las localidades rurales periféricas, algunas de las cuales se han integrado ya como colonias urbanas. ${ }^{3}$ Por ello, resulta de particular interés reconocer las transformaciones que están ocurriendo en estos espacios y cómo los viven los actores rurales situados en ellos.

Elobjetivo de la presenteinvestigación fue identificar las transformaciones que el crecimiento urbano de una ciudad media como San Cristóbal de Las Casas imprime sobre la actividad agrícola y las estrategias económicas de las familias situadas en su entorno rural, así como analizar las interacciones urbano-rurales que favorecen el desarrollo de los sistemas agrícolas periurbanos en beneficio de la población rural y urbana.

\section{Área de estudio}

Esta investigación se centró en un fragmento del espacio periurbano de San Cristóbal a fin de profundizar cualitativamente en las transformaciones locales que han ocurrido ante el crecimiento urbano. Una de las áreas boscosas y agrícolas más representativas de San Cristóbal es el cerro Huitepec, que alberga fragmentos de bosque mesófilo - en su mayoría dentro de dos áreas naturales protegidas-, situado a escasos seis kilómetros del centro de la ciudad. El trabajo se desarrolló en la ladera este del Huitepec, la cual está orientada a la ciudad, en un área de unas 1400 hectáreas donde están asentadas ocho localidades rurales que pertenecen administrativamente al municipio de San Cristóbal (ver mapa 2). De estas localidades, seis se segregaron entre 1970 y 2005 de una sola localidad antigua denominada El Huitepec, cuya presencia en la zona se remonta al menos a finales del siglo XIX -hoy son las localidades Alcanfores, Vistahermosa, Ocotal 1, Ocotal 2, Las Palmas y Santa Anita-; dos localidades más fueron fundadas después de 1980 por indígenas tsotsiles del vecino municipio de Chamula que salieron de su comunidad por motivos religiosos -Selva Natividad l y 2- (Calderón et al., 2012). En las últimas décadas, la población de estas localidades se ha incrementado notablemente, sobre todo a partir del año 2000, pasando de 1500 habitantes ese año a $2536^{4} \mathrm{en}$ 2010, lo que representa un crecimiento del 6.8\% anual (INEGI, 2010). Este crecimiento corresponde tanto al crecimiento natural de la población como a la llegada de pobladores urbanos a las localidades.

En esta zona también se ha presentadounincremento en los servicios urbanos - caminos, energía eléctrica, escuelas, transporte y, en algunas localidades, servicios telefónicos-, lo que facilita la comunicación con la ciudad (Calderón et al., 2012). Ante ello, el Huitepec se ha convertido en uno de los polos más demandados en el mercado urbano de tierras por sus atributos ambientales y estéticos, la cercanía a la ciudad, la propiedad privada de la tierra y la presencia de servicios públicos. El precio de la tierra se ha incrementado al grado de adquirir, en algunas zonas, un valor económico equiparable al del centro histórico de la ciudad, lo cual exacerba los procesos de cambio.

Son numerosas y diversas las transformaciones que han ocurrido en las localidades rurales, algunas de las cuales han sido expuestas en otros trabajos (Calderón et al., 2012; Zarate, 2008; García, 2005; Morales et al., en prensa). En este artículo abordamos la cuestión agrícola, pues constituye un reflejo de las transformaciones territoriales, culturales y económicas de la nueva ruralidad, aunque nos centramos sobre todo en sus modalidades y la relevancia económica que tiene para las familias.

\section{Métodos}

El trabajo de campo que sustenta esta investigación se realizó entre 2009 y 2010. Se realizaron 83 entrevistas estructuradas a pobladores de las localidades rurales 
del Huitepec, equivalentes al 15.8\% del total de las viviendas y más del 10\% de cada ranchería (cuadro 1). Los datos obtenidos incluyeron: a) información general de la unidad familiar - integrantes, ocupaciones, origen, etnicidad-; b) características de la propiedad y uso del suelo; c) fuentes de ingreso en la unidad familiar; d) en caso de haber producción agropecuaria, características de manejo, mercado y sus cambios en el tiempo. Además, se realizaron 17 entrevistas abiertas con informantes clave - autoridades locales y pobladores antiguos y recientes - para obtener información cualitativa de mayor profundidad sobre los cambios en la economía local, la agricultura y los actores sociales. También se realizaron recorridos de campo y observación participante en el Huitepec y algunos puntos de la ciudad.

La información disponible se capturó en una base de datos y se ordenó por localidades. Se realizó un análisis de contenido de las entrevistas organizando la información por categorías conceptuales con ayuda del programa Nvivo. Se realizaron análisis estadísticos descriptivos.

\section{Agricultura y diversificación socioeconómica en las localidades rurales}

Las localidades rurales del Huitepec presentan diferentes características en su conformación étnica, origen o propiedad de la tierra, algunas de las cuales se indican en el cuadro l. Sin embargo, en general en estas localidades la agricultura continúa siendo una actividad importante, sobre todo en términos culturales y de autoconsumo, ya que se realiza en más de la mitad de los hogares entrevistados (60.2\%), aunque en términos económicos contribuye sólo con un 17\% al ingreso familiar, con diferencias importantes entre las localidades (ver cuadro 2). Su relevancia económica es mayor en las localidades de Ocotal 1 y 2, Santa Anita y Las Palmas, que tienen población predominantemente nativa, con presencia indígena, disponen de terrenos de mayor tamaño y están más alejadas de la ciudad, donde la agricultura aporta entre el 30\% y el $44 \%$ al ingreso familiar (ver cuadros 1 y 2 ). Por otra parte, en las localidades Alcanfores y Vistahermosa, que tienen una mayor presencia de habitantes de origen urbano, son cercanas a la ciudad y poseen mayores servicios, la agricultura aporta menos del 10\%. En las localidades Selva Natividad l y 2, recientemente asentadas en el área y que disponen de terrenos pequeños, la agricultura tiene un aporte económico nulo.

La mayor parte de las familias estudiadas obtiene el grueso de sus ingresos monetarios (61.9\%) del sector terciario a través del empleo en el comercio u oficinas públicas en la ciudad de San Cristóbal, la realización de actividades profesionales o el ejercicio de oficios diversos —albañiles, jardineros, músicos, herreros-. El empleo en las propias localidades contribuye con un 9.2\% al ingreso promedio familiar en actividades como el comercio, el desempeño de algún oficio y el servicio doméstico en otras viviendas de la zona. Los programas de subsidio social, como el Programa Oportunidades, y los de apoyo a adultos mayores, contribuyen en casi el 12\%, y ninguna familia señaló recibir apoyos de algún programa de fomento productivo como Procampo. Por otro lado, sólo en Selva Natividad se reportó un caso de familiares migrantes que trabajan fuera del país.

Si consideramos las actividades que contribuyen al ingreso familiar y el papel de la agricultura, se pueden diferenciar entre la población de las localidades rurales del Huitepec cuatro sectores: a) familias con agricultura comercial, cuyos ingresos provienen en su mayoría de la agricultura (14\%); b) familias con agricultura intermedia, donde ésta contribuye parcialmente a los ingresos familiares (18\%); c) empleados urbanos con agricultura de autoconsumo, para quienes el aporte económico de la agricultura es nulo (28\%); y d) empleados urbanos sin actividad agrícola (40\%). Al considerar sólo a las familias con producción agrícola, el aporte económico de ésta se incrementa del 17\% al 28.4\% (ver cuadro 3), reduciéndose el ingreso a través del empleo.

\section{Características de la producción agrícola en el Huitepec}

La producción agrícola observada en el Huitepec corresponde en general a pequeños sistemas 
agroforestales en los que se presentan distintos tipos de cultivos: hortalizas, milpa, flores, frutas y hierbas aromáticas, siendo los más comunes los dos primeros.

Las hortalizas se cultivan en pequeñas superficies intensivas, generalmente cercanas a las viviendas, que cuentan con riego del agua procedente de manantiales locales. En estos huertos se cultivan diversas especies de hortalizas y se promueve la presencia de árboles frutales dispersos o barreras forestales. Se ha diferenciado en este trabajo entre hortalizas tradicionales - especies de consumo local poco demandadas en el mercado urbano- $\mathrm{y}$ hortalizas comerciales, que incluyen especies comunes en el mercado de San Cristóbal; a ellas, recientemente se han incorporado nuevas variedades de hortalizas demandadas por la población urbana de origen externo y por el turismo (ver cuadro 3).

El cultivo de maíz se realiza bajo el sistema de milpa tradicional, que consiste en la producción de maíz de temporal asociado con frijol, calabaza y otras herbáceas comestibles. La producción de flores se realiza de forma abierta $^{5}$ en superficies desmontadas o bajo sombra forestal y con escaso manejo. Tanto la milpa como las flores tienden a sembrarse en parcelas de mayor tamaño, que se sitúan en zonas altas e inclinadas del Huitepec alejadas de las viviendas. El tamaño del área sembrada tiene un promedio 2038 metros cuadrados, y puede ir desde pocos metros, en el caso de las familias que sólo siembran para su consumo hierbas aromáticas y otros productos de traspatio, hasta una hectárea y media para los productores que siembran milpa o que tienen cultivos comerciales de hortalizas y flores. Sin embargo, en general se trata de productores en pequeña escala aunque se observan importantes variaciones entre localidades (ver cuadro 3).

Más de la mitad de los agricultores (54\%) practica una producción "natural", que se riega con agua limpia y en la que no se utilizan insumos industrializados. En lugar de éstos, se recurre a los abonos naturales, al control manual de malezas y a los remedios naturales contra plagas y enfermedades. Un $38 \%$ combina esta producción con el uso de insumos químicos, en particular para el control de plagas y enfermedades. Sólo el 8\% utiliza insumos industriales también para fertilizar el suelo. Esto significa que, en su mayoría, los sistemas agrícolas tienen características que los podrían calificar como orgánicos, pero por carecer de un proceso de certificación formal no pueden usar esta denominación (Cámara de Diputados, 2006). ${ }^{6}$ La producción se dirige a distintos puntos de venta en la Ciudad de San Cristóbal, como los mercados municipales (65\%), un mercado solidario promovido por una iniciativa ciudadana llamado Tianguis de la Red de Productores y Consumidores Comida Sana y Cercana (13\%), y la venta directa a compradores particulares restaurantes o viviendas (13\%) -; el resto no especificó el lugar de venta.

Con respecto a la actividad pecuaria, un $76 \%$ de las familias posee animales domésticos de traspatio (ver cuadro 2), sobre todo aves, y otras crían ganado ovino, bovino y equino. La mayoría de las familias con producción pecuaria destina ésta al autoconsumo o al uso en la unidad doméstica ( $81 \%$ ), mientras que una menor proporción también la comercializa (16\%). Sólo en dos casos (3\%) las familias contaban con animales exclusivamente para fines comerciales.

\section{Transformaciones de la agricultura ante el crecimiento urbano}

El tipo de producción agrícola y pecuaria que encontramos actualmente en el Huitepec ha derivado de cambios graduales e históricos en los que ha influido su cercanía a San Cristóbal, de tal modo que en el momento actual reflejan una integración a la dinámica urbana. A continuación se indican, de manera muy sucinta, cuáles fueron las principales transformaciones indicadas en las referencias orales o documentales y los cambios producidos en los últimos 15 años señalados en las encuestas.

a) Disminución de ganadería extensiva e incremento de la producción intensiva de animales de corral

La producción de ganado ovino y equino en el valle de San Cristóbal fue sumamente común hasta antes de la década de los setenta del siglo pasado. A partir de esos 
años, como consecuencia del marcado crecimiento de la población, las tierras de uso pecuario disminuyeron drásticamente en la ciudad y se incorporaron al uso urbano (Ocampo, 2006). En el Huitepec existía una actividad ganadera en las partes bajas, pero también empezó a disminuir a causa de la parcelación de terrenos, como se indica en este testimonio:

\begin{abstract}
Antes pues era puro pastizal, no se miraban esos árboles como están ahorita, ya crecieron después. Cuando se destruyeron las vaquitas esas y empezó a crecer... Las teníamos en el cuarenta, sesenta, como en el setenta se acabó. Como ya se abundó la gente, ya no había dónde poner los animalitos. Como ya a mis hermanas les dio su parte mi papá, ya empezó a crecer las familias, por eso ya se fueron acabando. iTodo allá, aquello, era puro ganado, puro campo! (poblador nativo de Vistahermosa).
\end{abstract}

Actualmente, sólo algunas familias en la ranchería Ocotal, que disponen de amplios terrenos, poseen ganado bovino. En cambio, la cría de aves de corral, conejos, cerdos y otros animales que se pueden mantener en el pequeño traspatio es más generalizada. Estos cambios en el tipo de actividad pecuaria coinciden con una disminución en el tamaño de las propiedades y de las áreas productivas en las rancherías.

\section{b) Declive del cultivo de maíz e incremento en el cultivo de hortalizas}

El cultivo de maíz también fue una actividad de importancia en la zona hasta mediados de los cincuenta del siglo pasado; se realizaba en parcelas pequeñas para siembra de autoconsumo y en terrenos arrendados a los grandes propietarios de la zona. Aunque el cultivo de hortalizas parece haber sido una actividad antigua, a mediados del siglo XX se fue consolidando como la actividad agrícola con mayor relevancia económica ante la disminución del tamaño de las parcelas y una creciente demanda de vegetales en el núcleo urbano. Cabe señalar que la tierra fértil de origen volcánico del Huitepec - a diferencia del suelo calizo de la región-, la disponibilidad de agua y la presencia de zonas boscosas para la obtención de abono, hacen posible que esta actividad se realice con buenos resultados.

En los cincuentas eran milpa, como para los setentas fue que vendieron. Cuando yo tenía 14 años, como el 195l, hacíamos milpa, nos daban rentada la tierra y pagaban con una o dos fanegas de maíz, por eso tenía que ser bien trabajado, para que diera suficiente (pobladora de Alcanfores, 2009).

Cuando me casé llegué aquí con mi esposo. Antes sembraba milpa, después empecé con las hortalizas, pero sólo rábano y acelga sembrábamos. Ya luego fui sembrando más cosas (pobladora de Santa Anita, 2010).

Actualmente, el cultivo de maíz se ha reducido considerablemente; muchas familias abandonaron esta actividad y otras la transformaron hacia un uso más intensivo del suelo. En los últimos 15 años, un 20\% de las personas que aún realizan actividad agrícola redujo el tamaño del área cultivada o cambió el tipo de producción de milpa hacia hortalizas o flores. El motivo por el cual se redujo el área de cultivo consistió principalmente en la falta de espacio por la construcción de viviendas o por la división de los terrenos entre los miembros de la familia, y el cambio en el tipo de producción obedeció a la falta de fuerza de trabajo y a la baja rentabilidad del maíz en relación con los nuevos cultivos. Con respecto a las familias que han abandonado la agricultura, la principal razón fue la falta de fuerza de trabajo familiar por la pérdida de interés de los jóvenes en esta actividad ante la oportunidad del empleo urbano y el acceso a la educación.

\section{c) Incremento de las hortalizas y cultivos especializados ante la reorientación de los mercados urbanos}

La producción hortícola es importante en la región y se realiza también en otras localidades rurales periurbanas de San Cristóbal y en los vecinos municipios de Chamula y Zinacantán. Sin embargo, el Huitepec ha gozado de una particular preferencia por consumidores 
de distintos sectores económicos de la ciudad debido a las características de sus sistemas productivos. ${ }^{7}$ Por otro lado, algunos productores introdujeron nuevas variedades de hortalizas poco comunes en el mercado local a partir de la demanda de pobladores de origen externo. Esto propició que desde 2005, cuando surgió un mercado de productos de tipo orgánico en la ciudad, algunas familias del Huitepec fueran las principales proveedoras de productos hortícolas. Poco a poco, esta iniciativa ha ido evolucionando hasta constituir un pequeño mercado especializado donde productores y consumidores que pertenecen a la Red Mexicana de Tianguis y Mercados Orgánicos están realizando un proceso de certificación participativa de la calidad de los productos agrícolas del Huitepec. Esta Red define la certificación participativa como "un proceso colectivo entre productores, consumidores y otros actores, que garantiza la calidad orgánica y sana de productos locales, generados a pequeña escala, basado en relaciones de confianza y que promueven los compromisos de salud, ecología, equidad y certidumbre ambiental" (RMTPO, 2013).

Estas ventajas han significado un repunte en la actividad agrícola del Huitepec; los resultados muestran que, en los últimos 15 años, el 12\% de las familias amplió la superficie agrícola, y el 29\% de familias — que no cultivaba- inició esta actividad, mientras que el 6\% cambió su producción hacia variedades comerciales —es decir, pasaron de milpa a hortalizas o flores-. Las razones para estos cambios se encuentran en que los nuevos cultivos generan mayores beneficios económicos, pueden ser cultivados en superficies más pequeñas - hortalizas - o requieren menos trabajo -flores - en comparación con el maíz.

\section{d) Incremento en el mercado de tierras: fragmentación de las propiedades y participación de población urbana en la dinámica rural}

El desarrollo de los procesos de urbanización y del mercado de tierras en la zona ha tenido numerosos efectos locales. De todos ellos, queremos resaltar dos por su importancia en las transformaciones agrícolas del Huitepec. El primero se refiere a la compra-venta de terrenos, que ha propiciado la fragmentación del espacio y la reducción cada vez mayor del tamaño de las propiedades en las localidades rurales; en el momento de realizar esta investigación, el tamaño de los terrenos iba desde 200 metros cuadrados hasta cinco hectáreas, ${ }^{8}$ aunque la superficie más comúnmente observada era de 300 metros cuadrados (ver cuadro 1). Sin embargo, existe un proceso continuo e irregular de compra y venta de terrenos en la zona en el que han participado los agricultores y pobladores locales, aunque no necesariamente han sido ellos los más beneficiados, ya que también participan en este mercado numerosos intermediarios cuyo fin es la reventa y la obtención de ganancias económicas. Este fenómeno ha afectado con mayor intensidad a las localidades cercanas a la ciudad, y en menor medida a aquellas que tienen un perfil más rural e indígena, aunque no están fuera de este mercado. Los siguientes testimonios describen algunos rasgos de ésto:

Sí, hay como diez lotes que se han vendido en la colonia, a gente de Tuxtla y San Cristóbal. Los venden por emoción del dinero (pobladora de Santa Anita, 2010).

Mucha gente que ha llegado aquí es gente de Tuxtla, que tiene una casa de campo y que la visita tres veces al año, a veces una vez al año o no sé, para todo el año. Es gente que quiere un terreno grande, que quiere tranquilidad [...] Otro fenómeno que se está presentando, de mucha gente indígena que viene a esta zona y buscan terrenitos de 200 metros para ponerse a vivir. Pero en general en Alcanfores el [comprador de mayores ingresos] es el que viene, por el valor que tiene el terreno. Tal vez por otro lado [del Huitepec] sí sea más barato el terreno, pero aquí en Alcanfores no, está muy bien cotizado. Porque tenemos drenaje, agua, luz, la cercanía a San Cristóbal (poblador de Alcanfores, 2010).

El segundo efecto de la urbanización sobre la actividad agrícola que resaltamos se refiere precisamente a la multiplicación de actores sociales, con muy diversos 
perfiles, en la definición del uso del suelo en el Huitepec. La llegada de pobladores urbanos ha resultado conflictiva para los agricultores por factores como el uso del agua, ya que ésta proviene de los manantiales locales y los sistemas colectivos de distribución ya no alcanzan para cubrir en su totalidad a la población que la demanda. Otro aspecto que resulta interesante resaltar es que, entre los nuevos pobladores, el ideal de un modo de vida rural y campestre ha influido en un uso del suelo que favorece la presencia de espacios verdes y arbolados, algunos derivados de las áreas forestales y agrícolas previas. No son pocas las familias de origen urbano que, más allá del aspecto estético, incursionan activamente en el cultivo de hortalizas, la cría de animales de corral y el desarrollo de tecnologías alternativas, conformando un sector neo-rural que parece ir en ascenso en la zona.

\section{Expectativas de los habitantes del Huitepec hacia la agricultura}

Con respecto a las expectativas que las familias tienen sobre la actividad agrícola, más de la mitad de los entrevistados (54\%) piensa que esta actividad tiene viabilidad a largo plazo puesto que consideran que sus hijos o nietos podrán mantenerla en un futuro. Otros (18\%) creen que esta actividad sólo puede persistir a mediano plazo ya que es poco probable que sus hijos la retomen, pues están estudiando o no les interesa. El 14\% percibe problemas para continuar a corto plazo debido a la falta de tierra o tiempo, y un porcentaje similar no respondió. Por otro lado, algunas de las familias que no siembran actualmente tienen la expectativa de que sus hijos aprendan esta actividad y puedan dedicarse a ella en el futuro.

\section{Discusión}

Existe un consenso en cuanto a considerar que el creciente papel de las fuentes diversificadas de ingresos en las familias rurales y la disminución de la actividad agrícola son algunas de las características más sobresalientes de la nueva ruralidad (Carton de
Grammont, 2009; Kay, 2009). No obstante, conviene recordar que la economía campesina siempre se ha complementado con diversas fuentes, aunque son los cambios cualitativos y cuantitativos tan profundos que se presentan en la actualidad lo que se considera nuevo (Arias, 2009). En Los Altos de Chiapas, las abruptas características físicas y orográficas de la región constituyen una limitante para la producción agrícola, por lo que ésta se complementaba con la producción de ganado ovino y de animales de traspatio, la extracción forestal comercial y doméstica, la producción artesanal y manufacturera, las actividades comerciales y la venta de fuerza de trabajo en la ciudad de San Cristóbal o en las fincas de la región. Estos elementos configuraron los componentes de un modo de sobrevivencia campesina propio de la región en el que la agricultura tenía un papel central e incluso era subsidiada por el resto de las actividades (Parra y Mera, 1989). Hoy, las localidades rurales de la región han mantenido esa estructura diversificada en la economía familiar como parte de sus estrategias de vida, entre las cuales persiste el componente agrícola, aunque con una participación económica cada vez menor, y con un incremento de la participación del trabajo asalariado en el centro urbano y la dependencia de fondos provenientes de subsidios sociales o de la migración laboral nacional e internacional (Román, 2010; Martínez, 2006).

Como se observa en los resultados de este trabajo, las localidades del Huitepec situadas en los márgenes de la ciudad aún mantienen los componentes agrícolas en su economía, aunque más de la mitad del ingreso familiar proviene del empleo urbano, con una participación creciente de los servicios a terceros en la propia localidad ante la diversificación económica de sus habitantes. En el Huitepec, el 17\% de los ingresos familiares proviene de la agricultura y el $71 \%$ se obtiene del empleo en la ciudad o el autoempleo local. Por otra parte, el porcentaje de los ingresos familiares reportados a nivel nacional para las localidades rurales en 2004 era del 10\% y del 70\% respectivamente (Carton de Grammont, 2009). Es decir, en el Huitepec el ingreso económico a través del empleo es similar al promedio nacional, pero el aporte económico de la agricultura es más alto, lo cual puede 
reflejar la demanda de productos agrícolas para un mercado urbano.

Ahora bien, la participación porcentual de los subsidios de gobierno en el ingreso de las familias del Huitepec (12\%) es mayor a la reportada a nivel nacional para las localidades rurales (6\%), pero mucho menor a la que se ha encontrado en localidades rurales de la región - casi el 40\% en algunas de ellas- (Román, 2010; Carton de Grammont, 2009). Esto puede indicar la presencia diversa de programas sociales en el medio rural de esta región de Chiapas y el hecho de que, en una región tan empobrecida, el ingreso por los subsidios sociales y los apoyos productivos representen una alta proporción de la economía familiar. Se puede subrayar también la ausencia de remesas en los ingresos de las familias del Huitepec, lo que pudiera deberse al trabajo en la ciudad, que evitaría la migración laboral a otras regiones - como ocurre en localidades y municipios cercanos-, ante el aumento de la demanda de servicios y el creciente turismo en una ciudad considerada "pueblo mágico", con una significativa población inmigrante (Wilson, 2008). Se constata así que los lugares cercanos a los centros urbanos ofrecen nuevas opciones de pluriactividad para complementar los ingresos agrícolas de las familias con los obtenidos de distintos empleos en la ciudad, lo que representa una cualidad periurbana de la nueva ruralidad (Arias, 2009; Benítez, 2006; Torres et al., 1994).

Ahora bien, la población urbana de San Cristóbal también ha dependido históricamente de su entorno inmediato y regional para satisfacer sus necesidades alimenticias y de otros recursos, aun cuando dentro de los límites de la ciudad también ha existido una relevante actividad agrícola de traspatio. En el entorno antiguamente más inmediato a la ciudad, que ahora está conformado por colonias urbanas bien establecidas, se ubicaban haciendas cuya producción agrícola y animal se destinaba al consumo urbano (Pedrero, 1984). Más allá de los límites del valle, los pueblos indígenas de la región proveían de diversos productos y mano de obra a las familias urbanas, aunque en una relación desigual y excluyente. El crecimiento urbano y la inmigración regional de la población indígena circundante, que ha reproducido en la periferia de la ciudad sus sistemas agrícolas y otros elementos socioculturales de su región (Rodríguez, 2006), ha rebasado el límite que estableció la ciudad de San Cristóbal con su entorno, configurando un espacio periurbano donde las fronteras entre lo urbano y lo rural están fusionadas y son confusas (Ávila, 2004; Calderón et al., 2012). En términos de uso de suelo, esta fusión se manifiesta en un paisaje agroforestal heterogéneo, característico de las áreas rurales de la región, que a pesar de su condición periurbana todavía mezcla áreas forestales y agrícolas con distintas formas de urbanización (Calderón, 2012; Figueroa, 2011; Morales, en prensa).

Para la actividad agrícola, esta dinámica periurbana implica tanto oportunidades, como riesgos: las primeras están dadas por el mercado que ofrece la población urbana para los productos agrícolas, la facilidad de transporte de sus productos o la posibilidad que tienen las familias para alternar trabajo agrícola con otras fuentes de empleo. Por otra parte, el principal riesgo se manifiesta en la presión que representa la alta valorización económica de la tierra en el mercado inmobiliario frente al limitado ingreso económico de las actividades agrícolas (Aguilar y Escalona, 2000; Ávila, 2009; Torres y Rodríguez, 2008).También se puede señalar el cambio cultural que representa la cercanía a la ciudad, que propicia que muchos jóvenes de la zona prefieran estudiar y emplearse en otras actividades, dejando las agrícolas principalmente a cargo de hombres y mujeres de mayor edad, en especial en las familias que no tienen una clara orientación comercial.

La permanencia y eléxito de la agricultura como una actividad económica rentable en espacios periurbanos, en buena medida están determinados por la reducción de la agricultura de autoconsumo y por el cambio hacia productos destinados al mercado urbano (Torres y Rodríguez, 2008; Benítez, 2006; Aguilar y Escalona 2000), yen particular hacia la horticultura, que se ha expandido en numerosas ciudades, sobre todo en los países en desarrollo (Ávila, 2004). En el área periurbana del Huitepec ha ocurrido una transición de la producción de milpa a la horticultura o la floricultura, pero en este caso, a diferencia de otros espacios agrícolas en la misma 
ciudad de San Cristóbal, el esquema de producción limpia brinda un valor agregado que es aprovechado por los agricultores para ingresar a mercados especializados que les reportan mayores beneficios económicos. Sin embargo, son una opción para muy pocos productores por su reducido tamaño.

En el caso del pequeño mercado solidario que se ha desarrollado en la ciudad de San Cristóbal, el cual valora las condiciones de producción en la zona - comida sana - y busca disminuir los costos ambientales, económicos y sociales de traer alimentos desde lugares lejanos — comida cercana-, éste se presenta en un esquema de "agricultura apoyada por la comunidad", agricultura que va más allá del concepto de lo orgánico puesto que busca la reconstrucción de los sistemas alimentarios en su contexto socioecológico (Nelson et al., 2009). Sin embargo, los consumidores de este mercado hasta ahora han estado ligados a un sector social encabezado por población originaria de otras regiones; se estima que el tianguis tiene una afluencia de 100 a 130 familias consumidoras que se asocian a mayores ingresos económicos y a un nivel educativo más alto que los consumidores que asisten a los mercados municipales o los supermercados (Gutiérrez et al., 2012); los consumidores tienen diversas valoraciones ambientales y sociales sobre los productos que encuentran en este espacio, y consideran que con su compra están apoyando un proyecto alimentario que es bueno en términos de salud y medioambiente y que al mismo tiempo es más justo para los productores (Gutiérrez et al., 2012). Sin embargo, este mercado tiene un papel marginal tanto para comercializar la producción agrícola del Huitepec, de donde apenas participan algunas familias, como para representar un modelo viable de seguridad alimentaria urbana en las condiciones actuales. Los precios más altos de los productos agrícolas limpios, aunados a un limitado poder adquisitivo y al escaso conocimiento sobre este tipo de productos y mercados para la mayoría de la población urbana, hacen que las posibilidades de escalamiento sean reducidas. De esta forma, este tipo de experiencias aún se sitúa en un punto medio entre la construcción de formas alternativas de relaciones urbano-rurales y un mercado orientado a un sector de élite (Donald y Blay-Palmer, 2006). Por ello, en términos alimentarios, sobre todo, debe resaltarse el papel que la producción agropecuaria periurbana tiene para el autoconsumo de la población que la genera.

La permanencia de la agricultura urbana y periurbana tiene un papel fundamental como fuente de alimentos — maíz, hortalizas y frutas - para las familias productoras en la ciudad de San Cristóbal y como parte de la identidad cultural de los inmigrantes indígenas de la región (Rodríguez, 2006). A nivel mundial se ha observado que la agricultura continúa siendo una fuente de alimentos importante para las familias urbanas y periurbanas, y que constituye una parte fundamental para mejorar las condiciones de vida de los más pobres (Betancor y Modrego, 2011; Zezza y Tasciotti, 2010; Bryld, 2002; Altieri et al., 1999). Sería conveniente valorar el aporte que los diversos productos de traspatio significan para la economía familiar por la vía del ahorro, así como analizar su función como fuente de empleo local y profundizar en el papel que las actividades agropecuarias a pequeña escala tienen para las familias periurbanas del Huitepec.

Debido a todo lo anterior, los espacios en los que se desarrollan la agricultura urbana y periurbana deberían formar parte de los procesos de planificación del desarrollo urbano, pero más bien tienden a ser vistos como zonas de reserva para el crecimiento urbano y a valorarse únicamente en función de su valor inmobiliario (Ávila, 2004). Un mayor reconocimiento de la existencia de la agricultura en el medio urbano, así como de sus funciones y beneficios ambientales y sociales, abriría las posibilidades de regular e impulsar esta actividad en beneficio de la población urbana en general y en particular para los sectores de menos ingresos (Bryld, 2002).

\section{Conclusiones}

La integración del Huitepec en el centro urbano de San Cristóbal ha implicado la transformación de los sistemas productivos de ganadería, milpa, hortalizas y bosque hacia la producción de hortalizas y flores 
destinadas a un mercado urbano. Aunque muchas familias abandonaron la agricultura ante el crecimiento urbano, esta actividad aún tiene un papel relevante como parte de su consumo familiar y, no obstante el crecimiento urbano, reproduce una forma diversificada y heterogénea del paisaje en el espacio periurbano. De manera casi contradictoria, la persistencia y renovación de la agricultura en el Huitepec están ligadas a la misma ciudad, la cual brinda un mercado para los productos hortícolas locales y otras fuentes de ingreso a los agricultores. La emergencia de un mercado solidario urbano que integra aspectos de sustentabilidad y justicia ambiental y social representa una ventaja para la producción agrícola, pero no es garantía de su permanencia. Ante el mercado de tierras y el escaso control municipal, son los actores locales, de origen urbano o rural, los que en función de sus perspectivas y posibilidades pueden definir el uso futuro de la tierra periurbana.

El reconocimiento de estas nuevas formas de ruralidad, que exponen fuertes vínculos con el medio urbano, en una ciudad media como San Cristóbal, brinda otros referentes para el desarrollo de políticas públicas municipales o de procesos ciudadanos de desarrollo local a partir de una mayor y más armoniosa vinculación urbana y rural en un espacio específico.

\section{Notas}

${ }^{1}$ La primera autora agradece al CONACyT la beca de doctorado otorgada. El trabajo de campo se financió con recursos del Fondo Institucional de Fomento Regional para el Desarrollo Científico, Tecnológico y de Innovación de CONACYT, dentro del proyecto "Innovación socioambiental para el desarrollo en áreas de alta pobreza y biodiversidad de la frontera sur de México" (116306).

2 Existe un amplio debate académico sobre los alcances y el desarrollo teórico de la nueva ruralidad: algunas autoras muestran una posición crítica, pues consideran que sólo se trata de nuevas condiciones de despojo y dominio a las que ha sido sometido el sector campesino (Appendini y Mazuera, 2008; Rubio, 2003); otros autores plantean que el reconocimiento de los nuevos escenarios constituye una posibilidad de generar un desarrollo territorial más equitativo (Schejtam y Berdegué, 2004); y algunos más consideran que, en última instancia, representa un enfoque novedoso que ha permitido evidenciar numerosos aspectos de la realidad rural latinoamericana que quedaban ocultos bajo los análisis económicos y sociológicos tradicionales de lo urbano y lo rural (Grammont, 2010; Kay, 2009; Lambí y Pérez, 2007).

${ }^{3}$ Es el caso de la localidad de San Felipe Ecatepec, que se integró como colonia urbana en los años ochenta.

${ }^{4}$ INEGI omite aproximadamente la mitad de las viviendas de la localidad de Alcanfores, que son consideradas como parte del área urbana.

${ }^{5}$ En otro lado del Huitepec, en la ladera orientada a Zinacantán, existe una producción intensiva de flores en invernaderos con alto uso de insumos químicos.

6 Cámara de Diputados (2006), "Ley de productos orgánicos”, en Diario Oficial de la Federación, 7 de febrero.

${ }^{7}$ Una parte de las hortalizas que llegan a los mercados procedentes de las localidades periurbanas de San Cristóbal es regada con las aguas residuales de la ciudad, mientras que en otros municipios aledaños tradicionalmente hortícolas se emplea una fuerte cantidad de insumos químicos con escaso control en su manejo.

${ }^{8}$ En el área de estudio aún existen algunas grandes propiedades privadas de entre 30 y 50 hectáreas, aunque no todas se reconocen como parte de las localidades rurales.

\section{Referencias bibliográficas}

Aguilar, Adrián Guillermo y Miguel Escalona Maurice (2000), "Expansión metropolitana de la ciudad de México y el ámbito rural de Texcoco", en Pablo Alberto Torres Lima (comp.), Procesos metropolitanos y agricultura urbana. México: Universidad Autónoma Metropolitana Xochimilco, pp. 87-102.

Altieri, Miguel A., Nelson Companioni, Kristina Cañizares, Catherine Murphy, Peter Rosset, Martin Bourque y Clara I. Nicholls. (1999), "The greening of 
the «barrios»: Urban agriculture for food security in Cuba", en Agriculture and Human Values, vol. 16, núm. 2, pp. 131-140.

Appendini, Kristen A. y Gabriela Torres-Mazuera (2008), ¿Ruralidad sin agricultura?: perspectivas multidisciplinarias de una realidad fragmentada, México: El Colegio de México-Centro de Estudios Económicos.

Arias, Patricia (2005), "Nueva ruralidad: antropólogos y geógrafos frente al campo hoy", en Héctor Ávila Sánchez (comp.) Lo urbano-rural, inuevas expresiones territoriales?, Cuernavaca: Universidad Nacional Autónoma de México-Centro Regional de Investigaciones Multidisciplinarias, pp. 123-160.

Arias, Patricia (2009), "La pluriactividad rural a debate", en Hubert Carton de Grammont y Luciano Martínez (eds.), La pluriactividad en el campo latinoamericano, Quito: FLACSO-Ecuador, pp. 171-206

Arriaga Cabrera, Laura, José Manuel Espinoza Rodríguez, Claudia Aguilar Zúñiga, Eduardo Martínez Romero, Leticia Gómez Mendoza y Eleazar Loa Loza (2000), Regiones terrestres prioritarias de México. México: CONABIO.

Ávila Sánchez, Héctor (2009), "Periurbanización y espacios rurales en la periferia de las ciudades", en Estudios Agrarios, Revista de la Procuraduría Agraria, núm. 41, pp. 93-123.

Ávila Sánchez, Héctor (2004), "La agricultura en las ciudades y su periferia: un enfoque desde la Geografía”, en Investigaciones Geográficas. Boletín del Instituto de Geografía, núm. 53, pp. 98-121.

Banzo, M., (2005). "Del espacio al modo de vida. La cuestión periurbana en Europa Occidental: los casos de Francia y España", en Héctor Ávila Sánchez (ed.), Lo urbano-rural, inuevas expresiones territoriales?, Cuernavaca: UNAM-Centro Regional de Investigaciones Multidisciplinarias, pp. 207-24l.

Bellet Sanfeliu, Carmen y Josep María Llop Torne (2002), "Las líneas de trabajo del programa UIA-CIMES: ciudades intermedias y urbanización mundial", en Las nuevas funciones urbanas: gestión para una ciudad sostenible, Santiago de Chile: Naciones Unidas/CEPAL-División de Medio Ambiente y Asentamientos Humanos, pp. $33-47$.
Benítez González, Oscar Gabriel (2006), "Estrategias de regionalización urbano-rural desde una sociedad local: potencial para el desarrollo endógeno", en Vuivera, Revista de Estudios Urbanos, Regionales, Territoriales, Ambientales y Sociales, vol. 8, núm. 1, pp. 33-62.

Betancor, Andrea y Félix Modrego (2011), Estrategias de sustento de los hogares rurales y su evolución. Análisis de cuatro países latinoamericanos, Santiago de Chile: Programa Dinámicas Territoriales Rurales-Centro Latinoamericano para el Desarrollo Rural.

Bryld, Erick (2003), "Potentials, problems, and policy implications for urban agriculture in developing countries", en Agriculture and Human Values, núm. 20, pp. 79-86.

Burns, Allan (2000), "Solares de Mérida, Yucatán: recursos de clases sociales y etnias yucatecas", en Pablo Alberto Torres Lima (ed.), Procesosmetropolitanos y agricultura urbana, México: Universidad Autónoma Metropolitana Xochimilco, pp. 87-102.

Carton de Grammont, Hubert (2010), "Nueva ruralidad: iun concepto útil para repensar la relación campo-ciudad en América Latina?", en Ciudades, núm. 85, pp. 2-6.

Carton de Grammont, Hubert (2009), "La desagrarización del campo mexicano", en Convergencia. Revista de Ciencias Sociales, vol. 16, núm. 50, pp.13-55.

Calderón, Araceli, Lorena Soto Pinto y Erin Estrada (2012), "Entre la conservación del bosque y el crecimiento de la ciudad: las localidades rurales en el espacio periurbano del Huitepec", en Estudios Demográficos y Urbanos, vol. 27, núm. 81, pp. 739-787.

Calderón Cisneros, Araceli (2012), Territorios periurbanos y conservación de áreas agrícolas y forestales en una ciudad media. El caso del Huitepec en San Cristóbal de Las Casas, Chiapas, (tesis de doctorado), El Colegio de la Frontera Sur, San Cristóbal de Las Casas, Chiapas.

Consejo Nacional de Población (CONAPO) (2005), Sistema Urbano Nacional, 2005, México: CONAPO. 〈http://www.conapo.gob.mx/es/CONAPO/Sistema urbano_nacional_2005> [29 de noviembre de 2011].

Cruz Rodríguez, María Soledad (2002), "Procesos urbanos y 'ruralidad' en la periferia de la zona metropolitana de la Ciudad de México", en Estudios Demográficos y Urbanos, vol. 17, núm. 1, pp. 39-76. 
Donald, Betsy y Alison Blay-Palmer (2006), "The urban creative-food economy: producing food for the urban elite or social inclusion opportunity?", en Environment and Planing, vol. 38, núm. 10, pp. 1901-1920.

Figueroa Jáuregui, M. Lourdes, L. Alicia Ibáñez Castillo, Ramón Arteaga Ramírez, J. Luis Arellano Monterrosas y Mario Vázquez Peña (2011), "Cambio de uso de suelo en la cuenca de San Cristóbal de las Casas, Chiapas, México", en Agrociencia, núm. 45, pp. 431-544.

García García, Antonio (2005), La gestión del agua en la cuenca endorreica de San Cristóbal de Las Casas, Chiapas, México, (tesis de maestría en ciencias), Universidad Autónoma Chapingo-Dirección de Centros Regionales Universitarios, México.

García Romero, Arturo (2002), "An Evaluation of Forest Deterioration in the Disturbed Mountains of Western Mexico City", en Mountain Research and Development, núm. 22, pp. 270-277.

Gómez E., Sergio (2002). La nueva ruralidad, qué tan nueva? : revisión de la bibliografía, un intento por definir sus límites y una propuesta conceptual para realizar investigaciones, Chile: Universidad Austral de Chile-Facultad de Filosofía y Humanidades.

Gutiérrez Pérez, Cynthia, Esperanza Tuñón Pablos, Fernando Limón Aguirre, Helda Morales y Ronald Nigh (2012), "Representaciones sociales de los alimentos orgánicos entre consumidores de Chiapas", en Estudios Sociales, vol. 20, núm. 39, pp. 100-129.

INEGI (2010), Censo de poblacióny vivienda 2010. Principales resultados por localidad, México: INEGI. 〈http:// www.inegi.org.mx/sistemas/consulta_resultados/ iter2010.aspx?c=27329\&es=est $>$ [25 de octubre de $2011]$.

Kay, Cristóbal (2009), "Estudios rurales en América Latina en el periodo de globalización neoliberal: una nueva ruralidad?", en Revista Mexicana de Sociología, vol. 71, núm. 4, pp. 607-645.

Lambí Insua, Luis y Edelmira Pérez Correa (2007), "Nuevas ruralidades y viejos campesinismos. Agenda para una nueva sociología rural Latinoamericana", en Cuadernos de Desarrollo Rural, núm. 59, pp. 37-61.

Martínez, Germán 2006, “Desarrollo regional, sociodemografía y condiciones de vida de la población Chamula, Chipas", en Papeles de Población, núm. 34, pp. 259-277.

Morales, Helda, M. Castillo, Ronald Nigh y E. Valencia (en prensa), "Conservación del Huitepec: mirando hacia afuera de la Reserva", en La diversidad biológica de Chiapas. Estudio de estado, México: CONABIO/ Gobierno del Estado de Chiapas, pp. 298-299.

Morello, J., S. D. Matteucci y A. Rodríguez (2003), "Sustainable Development and Urban Growth in the Argentine Pampas Region", en Annals of the American Academy of Political and Social Science, núm. 590, pp. 116130.

Nelson, Erin, Laura Gómez Tovar, Rita Schwentesius y Manuel Ángel Gómez Cruz (2009), "Participatory organic certification in Mexico: an alternative approach to maintaining the integrity of the organic label", en Agriculture and Human Values, núm. 27, pp. 227-237.

Ocampo Morales, Alberto Abraham (2006), Análisis de los sistemas de producción bovina periurbana del valle de San Cristóbal, (tesis de maestría), El Colegio de la Frontera Sur, San Cristóbal de Las Casas, Chiapas.

Parra, M.R. y L.M. Mera (1989), "La organización social para la producción", en El subdesarrollo agrícola en Los Altos de Chiapas, México: Universidad Autónoma de Chapingo, pp. 315-405.

Pedrero Nieto, Gloria (1984), "Las haciendas y los ranchos sancristobalenses del siglo XIX. Estudio histórico", en San Cristóbal y sus alrededores, Tuxtla Gutiérrez, Chiapas: Gobierno del Estado de Chiapas.

Pothukuchi, Kameshwari y Jerome L. Kaufman (1999) "Placing the food system on the urban agenda: The role of municipal institutions in food systems planning", en Agriculture and Human Values, vol. 16, núm. 2, pp. 213-224.

Ramírez, Blanca R. (2005), "Miradas y posturas frente a la ciudad y el campo", en Héctor Ávila Sánchez (comp.), Lo urbano-rural, inuevas expresiones territoriales?, Cuernavaca: UNAM-Centro Regional de Investigaciones Multidisciplinarias, pp. 61-85.

Ramírez, Blanca R. (2000), "Las dimensiones regionales de la relación campo-ciudad, el caso de Querétaro, México", en Pablo Alberto Torres Lima (ed.), Procesos 
metropolitanos y agricultura urbana, México: Universidad Autónoma Metropolitana Xochimilco, pp. 67-85.

RMTMO (2013), Certificación participativa, México: Red Mexicana de Tianguis y Mercados Orgánicos. 〈http:// tianguisorganicos.org.mx/certificacion-participativa/> [junio de 2013].

Rodríguez Galván, Guadalupe (2006), "La producción agropecuaria en la periferia de San Cristóbal de Las Casas, Chiapas", en Anuario de estudios indígenas XI, Chiapas: Universidad Autónoma de Chiapas-Instituto de Estudios Indígenas, pp. 249-272.

Román Ruiz, Sandra Ibeth (2010), Seguridad alimentaria con enfoque en modos de vida sustentable en el municipio de Oxchuc, Chiapas (tesis de maestría en ciencias en recursos naturales y desarrollo rural), El Colegio de la Frontera Sur, San Cristóbal de Las Casas, Chiapas.

Rubio, Blanca (2003), Explotados y excluidos: los campesinos lationoamericanos en la fase agroexportadora neoliberal, México: Plaza y Valdés/Universidad Autónoma de Chapingo.

Ruiz Rivera, Naxhelli y Javier Delgado (2008), "Territorio y nuevas ruralidades: un recorrido teórico sobre las transformaciones de la relación campo-ciudad", en EURE (Santiago), vol. 34, núm. 102, pp. 77-95.

Salazar Cruz, Clara E. (2000), "La relación entre la población y los recursos naturales en un área de expansión de la Ciudad de México", en Estudios Demográficos y Urbanos, vol. 15, núm. 44, pp. 287-324.
Schejtman, Alexander y Julio A. Berdegué (2004), Desarrollo territorial rural, Chile: RIMISP-Centro Latinoamericano para el Desarrollo Rural.

Torres Lima, Pablo Alberto y L. Rodríguez-Sánchez (2008), "Farming dynamics and social capital: A case study in the urban fringe of Mexico City", en Environment, Development and Sustainability, núm. 10, pp. 193-208.

Torres Lima, Pablo Alberto, Beatriz Canabal Cristiani y Gilberto Burela Rueda (1994), "Urban sustainable agriculture: The paradox of the chinampa system in Mexico City", en Agriculture and Human Values, vol. 11, núm. 1, pp. 37-46.

Velázquez Torres, David (2004), Barrio Primero de Enero, San Cristóbal de Las Casas, Chiapas, México: Secretaría de Desarrollo Social/CIESAS.

Wilson, Tamara Diana (2008), "Economic and social impacts of tourism in Mexico", en Latin American Perspectives, vol. 35, núm. 3, pp. 37-52.

Zarate Toledo, María Antonieta (2008), Gestión del agua y conflicto en la periferia urbana de San Cristóbal de Las Casas, Chiapas: El caso de los Alcanfores. San Cristóbal de las Casas, Chiapas, (tesis de maestría), Centro de Investigaciones y Estudios Superiores en Antropología Social, México. Zezza, Alberto y Luca Tasciotti(2010), "Urban agriculture, poverty, and food security: Empirical evidence from a sample of developing countries", en Food Policy, núm. 35, pp. 265-273. 
Cuadro 1. Localidades rurales del Huitepec, principales características

\begin{tabular}{|c|c|c|c|c|c|c|c|}
\hline Localidad & Población* & Viviendas* & $\begin{array}{c}\text { Encuestas } \\
\text { aplicadas } \\
\mathbf{N}\end{array}$ & Muestra\% & $\begin{array}{c}\text { Hablantes } \\
\text { indigenas } \\
* *(\%)\end{array}$ & $\begin{array}{c}\text { Familias } \\
\text { nativas } \\
* *(\%)\end{array}$ & $\begin{array}{c}\text { Tamaño } \\
\text { promedio de } \\
\text { propiedad } \\
* *\left(\mathbf{m}^{2}\right)\end{array}$ \\
\hline Alcanfores & 282 & 65 & 17 & 26.1 & 17.6 & 64.7 & 2024 \\
\hline Vistahermosa & 497 & 110 & 15 & 13.6 & 20 & 66.7 & 4808 \\
\hline Ocotal 1 & 291 & 60 & 9 & 13.30 & 55.6 & 88.9 & 7662 \\
\hline Ocotal 2 & 241 & 53 & 8 & 11.3 & 62.5 & 100.0 & 1243 \\
\hline Las Palmas & 269 & 60 & 8 & 16.6 & 12.5 & 87.5 & 7471 \\
\hline Santa Anita & 106 & 23 & 6 & 30.4 & 33.3 & 83.3 & 4016 \\
\hline $\begin{array}{l}\text { Selva } \\
\text { Natividad } 1\end{array}$ & 699 & 123 & 14 & 11.4 & 100 & 14.3 & 693 \\
\hline $\begin{array}{l}\text { Selva } \\
\text { Natividad } 2\end{array}$ & 151 & 29 & 6 & 20.7 & 100 & 100.0 & 608 \\
\hline Total general & 2536 & 523 & 83 & 15.8 & 46.9 & 68.6 & 3316 \\
\hline
\end{tabular}

Fuentes: INEGI (2010) y trabajo de campo 2010.

*Según INEGI (2010).

**Proporción en relación con la muestra encuestada.

Cuadro 2. Características generales de la producción agropecuaria y fuentes de ingreso en las localidades rurales del Huitepec

\begin{tabular}{|c|c|c|c|c|c|c|c|c|}
\hline \multirow[t]{2}{*}{ Localidad } & \multicolumn{2}{|c|}{ Practican agricultura } & \multicolumn{2}{|c|}{ Producción pecuaria } & \multicolumn{4}{|c|}{ Fuentes de ingreso familiar \% * } \\
\hline & (n) & $\%$ & (n) & $\%$ & Agrícola & $\begin{array}{l}\text { Empleo } \\
\text { urbano }\end{array}$ & $\begin{array}{c}\text { Empleo } \\
\text { local }\end{array}$ & $\begin{array}{c}\text { Subsidio } \\
\text { social }\end{array}$ \\
\hline Alcanfores & 10 & 58.8 & 13 & 76.5 & 5.3 & 65.3 & 12.4 & 17.1 \\
\hline Vistahermosa & 7 & 46.7 & 10 & 66.7 & 8.7 & 68.0 & 8.0 & 15.3 \\
\hline Ocotal 1 & 6 & 66.7 & 8 & 88.9 & 44.4 & 48.9 & 0.0 & 6.7 \\
\hline Ocotal 2 & 5 & 62.5 & 7 & 87.5 & 31.3 & 58.8 & 1.3 & 8.8 \\
\hline Las Palmas & 8 & 100.0 & 7 & 87.5 & 38.8 & 43.8 & 1.3 & 16.3 \\
\hline Santa Anita & 5 & 83.3 & 5 & 83.3 & 40.0 & 40.0 & 13.3 & 6.7 \\
\hline $\begin{array}{l}\text { Selva } \\
\text { Natividad } 1\end{array}$ & 8 & 57.1 & 12 & 85.7 & 0.0 & 75.0 & 15.0 & 10.0 \\
\hline $\begin{array}{l}\text { Selva } \\
\text { Natividad } 2\end{array}$ & 1 & 16.7 & 1 & 16.7 & 0.0 & 75.7 & 17.1 & 7.1 \\
\hline Total general & 50 & 60.2 & 63 & 75.9 & 17.1 & 61.9 & 9.2 & 11.8 \\
\hline
\end{tabular}

Fuente: trabajo de campo 2010.

*Porcentaje con el que contribuyen las distintas fuentes al ingreso total familiar. 


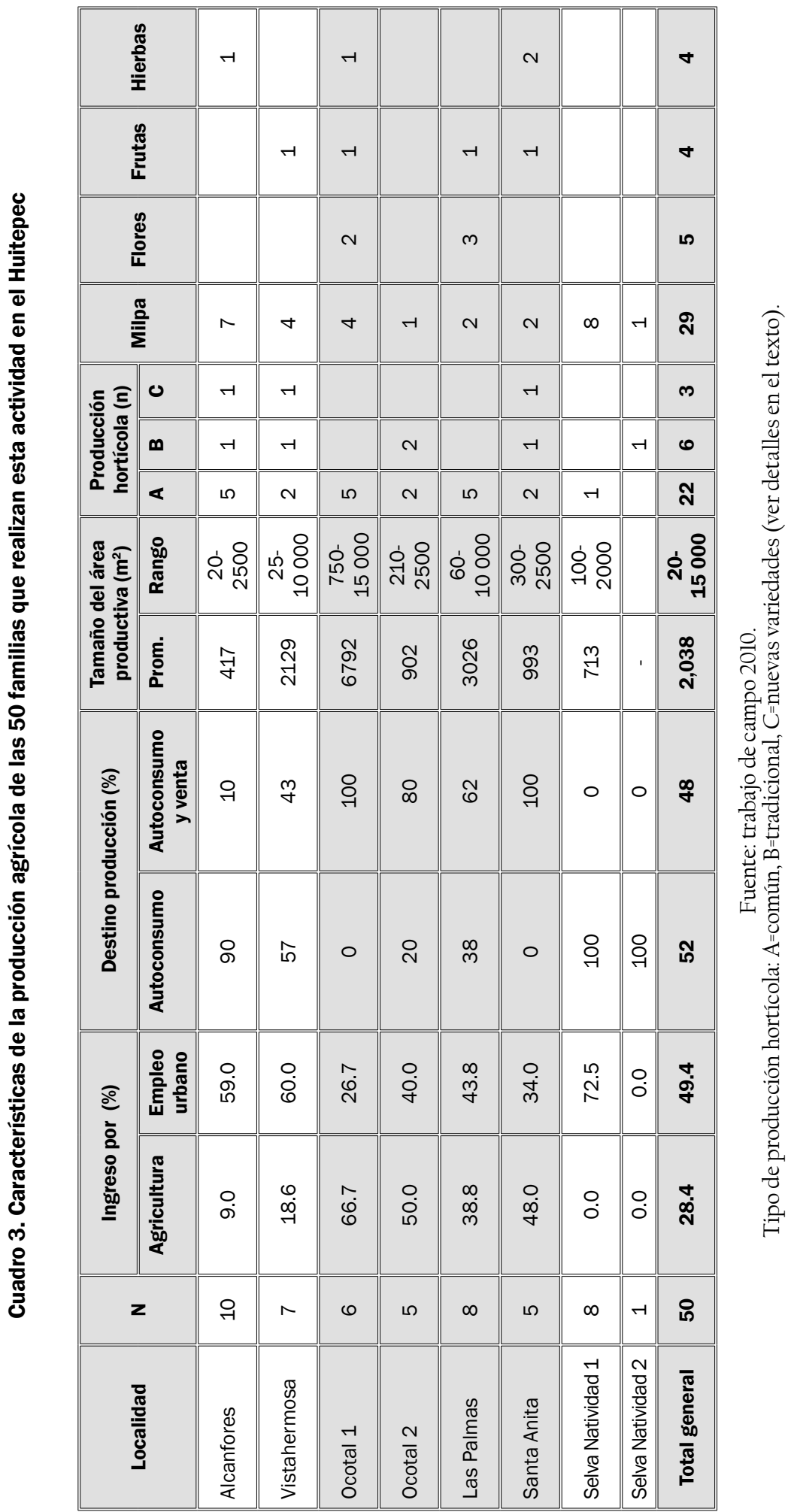


Mapa 1. Crecimiento del área urbanizada de San Cristóbal de Las Casas, Chiapas

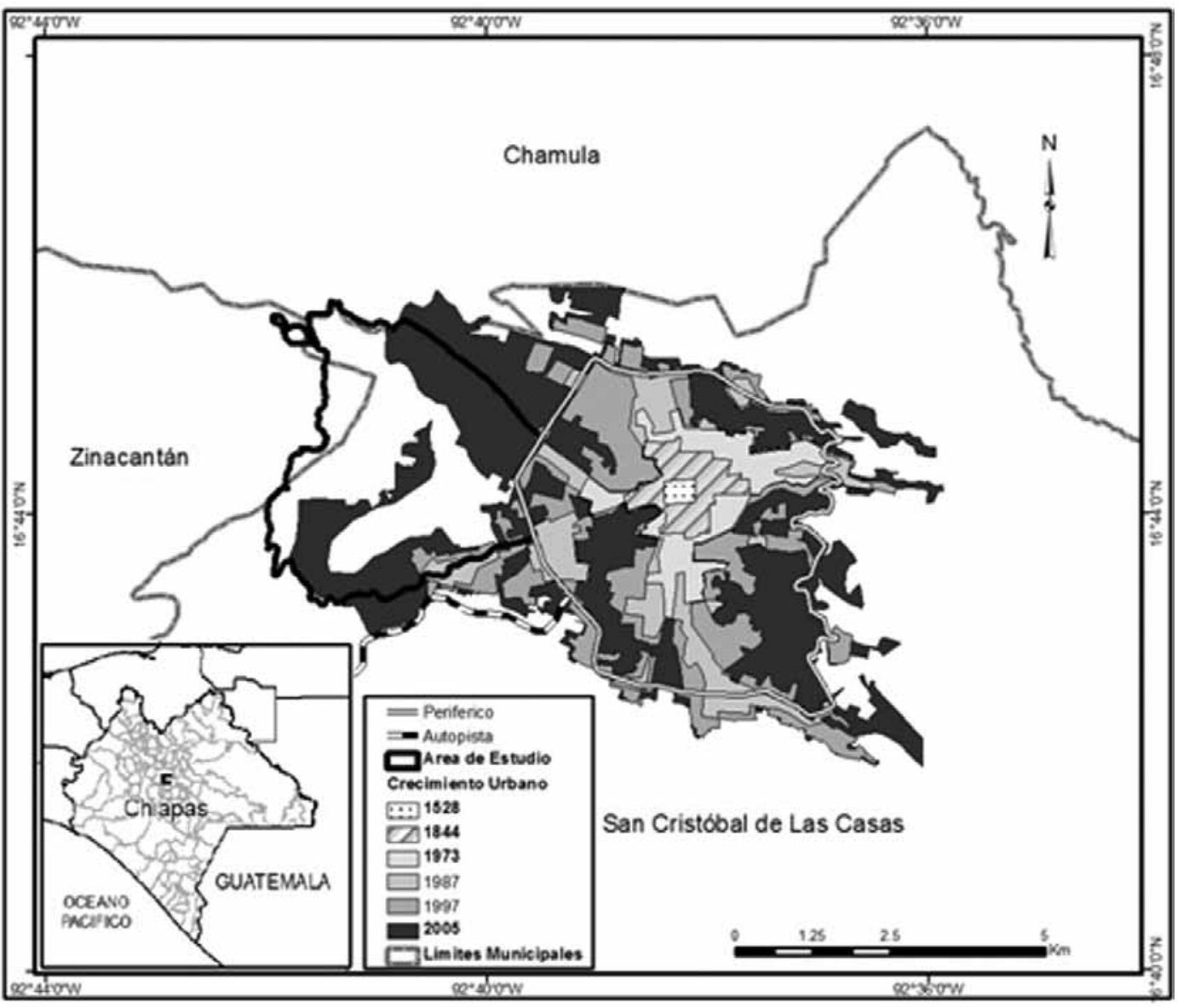

Fuente: Laboratorio de Análisis de Información Geográfica y Estadística (LAIGE), ECOSUR 
Mapa 2. Localidades rurales del Huitepec y traza urbana de San Cristóbal de Las Casas, Chiapas

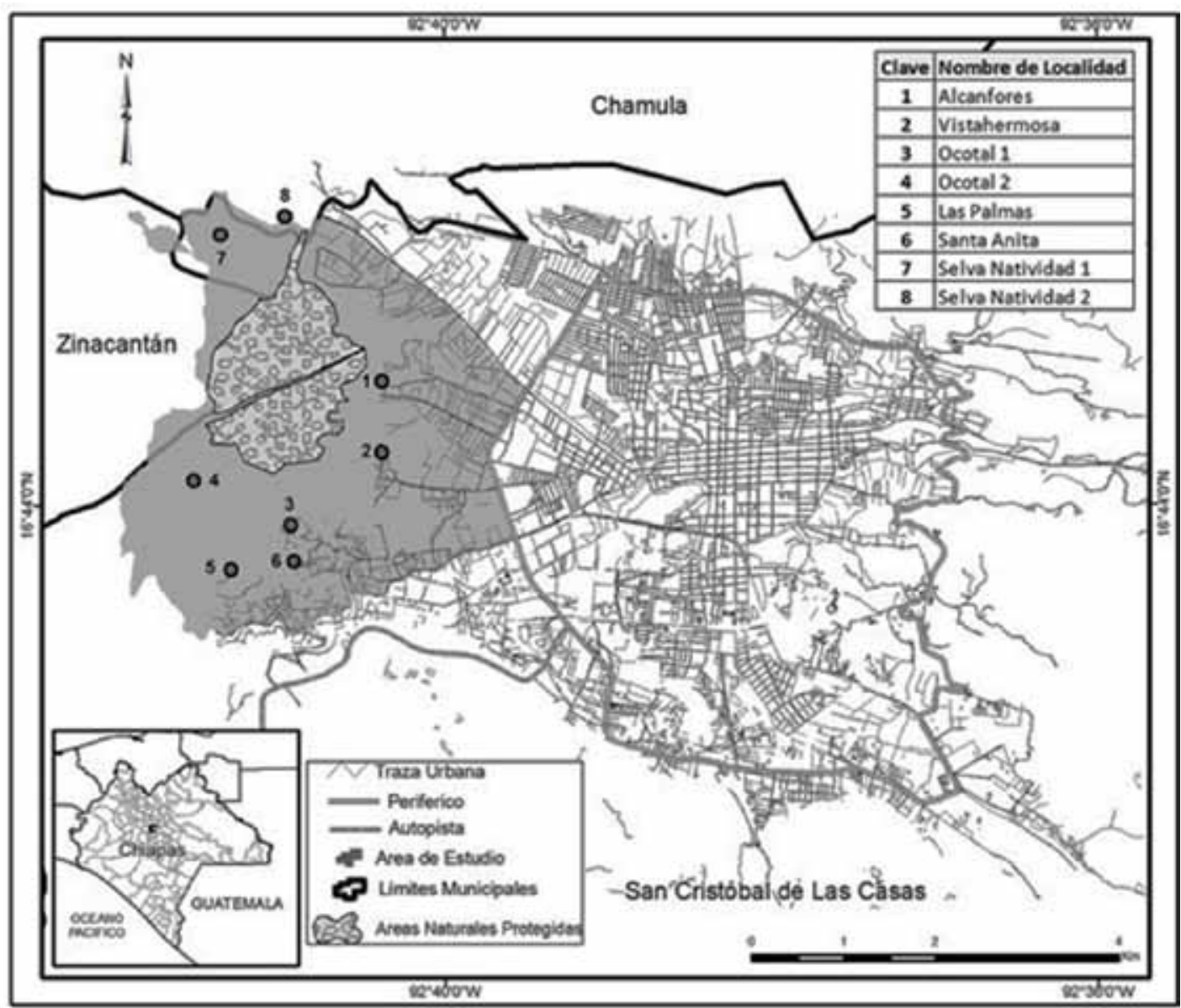

Fuente: Laboratorio de Análisis de Información Geográfica y Estadística (LAIGE), ECOSUR 